\section{Daniel Poulain \\ Christine Faille Jean-Claude Michalski Daniel Camus}

\title{
Anticorps monoclonaux anti-Candida albicans : de la biologie cellulaire aux marqueurs du comportement pathogène
}

La levure Candida albicans est un saprophyte habituel du tube digestif humain qui peut être à l'origine d'infections opportunistes, localisées ou généralisées, parfois sévères chez des malades affaiblis et immunodéprimés. La possession d'anticorps monoclonaux divers, principalement dirigés contre la copule glucidique de la paroi du micro-organisme, a permis de progresser dans l'étude de la biologie cellulaire et de sa variabilité antigénique. De plus, ces réactifs ont permis d'améliorer le diagnostic immunologique des formes pathogènes de candidose et pourraient être utilisés dans l'avenir comme sondes radiomarquées permettant la détection in vivo par scintigraphie de foyers profonds de l'affection.

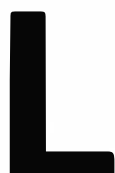

es candidoses sont, par leur fréquence et leur gravité, au premier rang des infections opportunistes. Leur diagnostic reste difficile et tés, praticien hospitalier, service de parasitologiemycologie du centre hospitalier universitaire de Lille. C. Faille : stagiaire post-doctoral. J.-C. Michalski : chargé de recherche Inserm, laboratoire de chimie biologique et unité associée au Cnrs 217, université des sciences et techniques de Lille Flandres-Artois, 59650 Villeneuve d'Ascq. D. Camus : professeur de parasitologie à l'université de Lille II, directeur de l'unité Inserm 42. Unité de biologie et de biochimie parasitaires et fongiques, Inserm U. 42, 369, rue Jules-Guesde, 59650 Villeneuve-d'Ascq, France.

TIRÉS A PART

D. Poulain. les mécanismes intimes régissant la transformation de levures banales en redoutables agents pathogènes sont toujours inconnus. L'interaction des composants de la levure avec le système immunitaire a suscité d'innombrables travaux. Depuis quatre années, l'utilisation, en ce domaine, d'anticorps monoclonaux a permis la caractérisation d'antigènes physiologiquement majeurs et fourni des sondes pour la détection de substances fongiques circulantes au cours des processus invasifs. Sur le plan fondamental, les anticorps monoclo- naux identifiant les glycoprotéines de la paroi permettent de mieux comprendre leur structure et leur biosynthèse. Ils démontrent la complexité de leur expression antigénique et son caractère fluctuant. Les mécanismes de cette variabilité conditionnent vraisemblablement les relations hôteparasite extrêmement subtiles de l'opportunisme.

Candida albicans est une levure susceptible de produire du mycélium, dont l'habitat naturel est le tube digestif humain. A la suite de causes favorisantes bien connues [1], cet organisme peut envahir tous les tissus superficiels ou profonds. Une dizaine d'autres espèces du genre Candida partagent à des degrés divers avec C. albicans ce statut de pathogène 
opportuniste. Les candidoses superficielles sont généralement bénignes mais volontiers récidivantes. Les candidoses profondes sont des infections essentiellement nosocomiales* dont l'incidence et la symptomatologie évoluent parallèlement aux progrès des techniques médico-chirurgicales. Elles sont une préoccupation constante de nombreux services cliniques, en particulier des services de chirurgie et de réanimation dans lesquels se manifeste la majorité des candidoses hospitalières [2]. L'état des patients hospitalisés dans ces services étant fragilisé, les facteurs de risque sont multiples : contamination massive d'origine endogène pouvant résulter d'une intervention sur la sphère digestive, contamination exogène par cathétérisme, drains, plaies, antibiothérapie à large spectre... Ainsi, alors que les techniques de transplantation d'organes ont permis d'améliorer considérablement le pronostic de nombre d'affections, les efforts de soins considérables mis en œuvre peuvent être anéantis par une infection déterminée par une levure banale [3]. Pour lutter contre les candidoses, des molécules antifongiques à visée systémique de plus en plus efficaces sont disponibles, mais leur toxicité interdit toujours leur emploi systématique. Il est donc essentiel d'établir un diagnostic précoce et d'entreprendre une thérapeutique spécifique. L'absence de signes cliniques spécifiques ajoutée au caractère ubiquitaire et opportuniste des agents infectieux font que, malgré de récents progrès, le diagnostic demeure difficile. Ces dernières années ont vu se développer l'utilisation d'anticorps monoclonaux dans le but d'obtenir a) des sondes spécifiques d'espèces ou de genres permettant une identification rapide de souches; b) des sondes spécifiques de souches pouvant être utilisées comme marqueurs épidémiologiques ; c) des anticorps dirigés contre des antigènes exprimés préférentiellement lors de la phase pathogène, permettant de les caractériser puis de les immunopurifier ; d) des anticorps permettant de détecter des antigènes circulants dans les

* Survenant en milieu hospitalier. $\mathrm{m} / \mathrm{s} n^{\circ} 6$ vol. 6 , juin 90

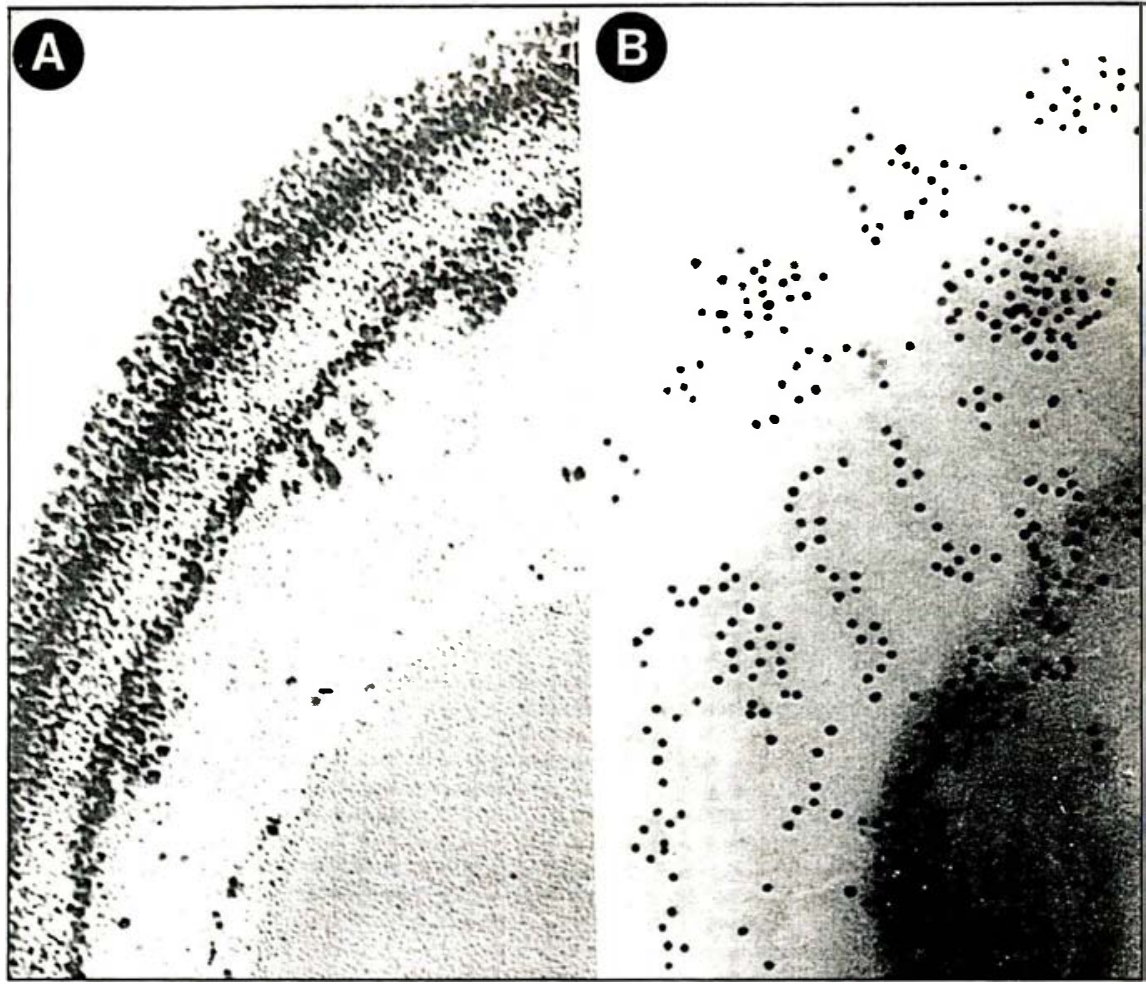

Figure 1. Micrographies de paroi de C. albicans, observée en microscopie électronique à transmission. A, méthode cytochimique de Thierry. Le contraste aux électrons est associé à la présence de polysaccharides sensibles à l'oxydation périodique (liaisons [1-6] et [1-2]), la présence d'une grande quantité de ces liaisons dans les phosphomannoprotéines pariétales amène à les localiser dans les couches externes. B, immunodétection ultrastructurale impliquant un anticorps monoclonal anti-mannoprotéines pariétales couplé à l'or colloïdal. La mise en place des antigènes à la surface s'opère par des voies de cheminement préférentielles au sein de la paroi.

sérums de patients atteints de candidoses systémiques. Ces travaux sont indissociables d'études plus fondamentales, dans lesquelles les anticorps monoclonaux ont été également impliqués. Il s'agit des recherches sur la biologie cellulaire des Candida et le déterminisme de leur pathogénicité. L'objectif de cet article est de dresser, au travers de notre expérience, un bilan de l'utilisation des anticorps monoclonaux dans les domaines fondamentaux et appliqués.

\section{L'identification de genres, d'espèces, de souches}

Des sérums hyperimmuns produits contre des levures tuées, adsorbés sur des espèces hétérologues ont pu conduire à une véritable classification sérologique des Candida [4]. La même démarche a permis l'identification de sérotypes au sein des espèces $C$. albicans et $C$. tropicalis [5]. Ces distinctions reposent sur la reconnaissance par les IgG de lapin d'antigènes thermostables de paroi. Les composés matriciels (figure 1), extractibles notamment par autoclavage, constituent le support de cette spécificité [6]. Ces extraits, décrits sous le terme de mannanes, sont en fait des molécules complexes de phosphopeptidomannanes (figure 3 , p. 529). Le raffinement des méthodes d'analyse immunochimique a permis d'identifier un hexaose des chaînes latérales des mannanes de sérotype A [7] 


\section{RÉFÉRENCES}

1. Odds FC. Factors that predispose the host to Candidosis. In : Odds FC, ed. Candida and Candidosis. London : Bailliere Tindall, 1988 : 93-104.

2. Poulain D, Ayadi A, Dutoit E, Arbon G. Le diagnostic biologique des candidoses post-opératoires. La Semaine des Hôpitaux $1989 ; 13$ : 795-800.

3. Les complications infectieuses des transplantations d'organes. La Lettre de l'Infectiologue. Numéro spécial $1989 ; 4: 275-322$.

4. Tsuchiya T, Fukazawa Y, Taguchi M, Kase T, Shinoda T. Serologic aspects on yeast classification. Mycopath Mycol Applicata $1974 ; 53: 77-85$.

5. Hasenclever HF, Mitchell WO. Antigcnic studies of Candida. I. Observation of two antigenic groups in Candida albicans. J Bacteriol 1961; 82 : 570-3.

6. Summers DF, Grollman AP, Hasenclever HF. Polysaccharide antigens of the Candida cell wall. J Immunol $1964 ; 92: 491-9$.

7. Fukazawa Y, Nishikawa A, Suzuki M, Shinoda T. Immunological basis of the serologic specificity of the yeast : immunochemical determinants of several antigenic factors of yeasts. In : Preuser HJ, ed. Medical Mycology. Stuttgart : Gustav Fisher, 1980 : 127-36.

8. Suzuki M, Fukazawa Y. Immunochemical characterization of Candida albicans cell wall antigens : specific determinant of Candida albicans serotype A mannan. Microbiol Immunol $1982 ; 26: 387-402$.

9. Chardes T, Piechaczyk M, Cavailles V, Salhi SL, Pau B, Bastide JM. Production and partial characterization of anti-Candida monoclonal antibodies. Ann Inst Pasteur/Immunol $1986 ; 137$ : 117-25.

10. Miyakawa Y, Kagaya K, Fukazawa Y, Soe G. Production and characterization of agglutinating monoclonal antibodies against predominant antigenic factors for Candida albicans. J Clin Microbiol 1986 ; 23 : 881-6.

11. Poulain D, Cailliez JC, Dubremetz JF. Secretion of glycoproteins through the cell wall of Candida albicans. Eur J Cell Biol 1989 ; $50: 94-9$.

12. Cailliez JC, Poulain D. Analyse cytologique de l'expression d'un épitope porté par les glycoprotéines excrétées par Candida albicans. Ann Microbiol (Inst. Pasteur) 1988 ; 139: 171-88.

13. Shibata N, Kobayashi $H$, Tojo M, Suzuki S. Characterization of phosphomannan-protein complexes isolated from viable cells of yeast and mycelial forms of Candida albicans NIH B-792 strain by the action of zymolyase 100-T. Arc Biochem

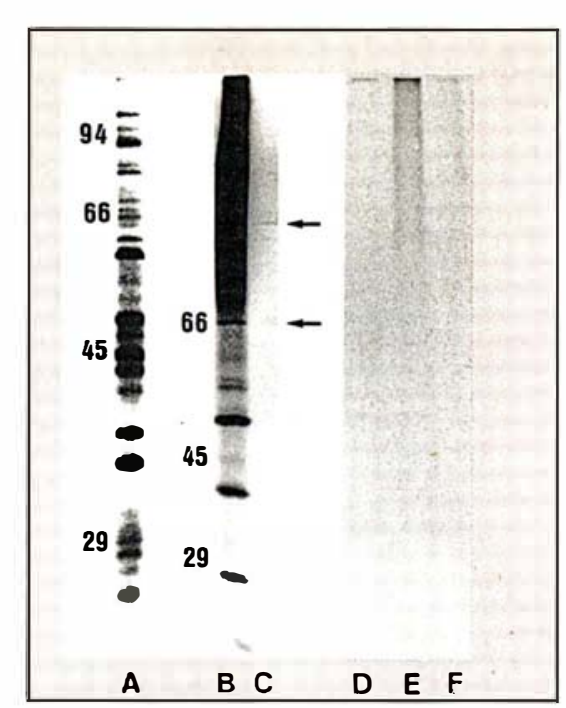

Figure 2. Profils électrophorétiques de protéines radiomarquées par incorporation de méthionine 355 . $A$, protéines cytoplasmiques. B, produits $d^{\prime}$ excrétion/sécrétion après culture brève en milieu synthétique, les glycoprotéines à copule polysaccharidique importante se présentent sous forme de trainées dans le gel de polyacrylamide. C, l'adjonction de tunicamycine au milieu de culture entraîne la disparition de ces copules, les produits excrétés se présentent alors sous forme de protéines bien résolues sur le gel. D, immunoprécipitation par un anticorps monoclonal identifiant la copule polysaccharidique d'une glycoprotéine excrétée.

(figure 3C), puis un heptaose [8] comme étant le support de cette spécificité sérologique. Cette conclusion résulte de l'analyse du pouvoir inhibiteur de différents fragments d'acétolyse (figure 3-2) envers la réaction de l'antisérum avec des mannoprotéines pariétales. La même démarche a été appliquée avec succès à l'identification de nombreuses spécificités sérologiques d'espèces ou de genres. La spécificité des anticorps polyclonaux monospécifiques est telle qu'il est possible, en les utilisant de manière séquentielle, d'aboutir à l'identification de souches sauvages par agglutination directe, un "kit " a d'ailleurs été commercialisé à cet effet. De même, la spécificité du sérotypage de $C$. albicans a permis le typage épidémiologique des souches, en relation notamment avec la résistance à la 5 fluorocytosine.

Dans ce contexte, l'obtention d'anticorps monoclonaux réagissant spécifiquement avec $C$. albicans ou, à un moindre degré, d'autres levures du genre Candida, fut un objectif partagé par de nombreuses équipes en vue de remplacer les méthodes longues et onéreuses d'identification, morphologiques ou physiologiques. Malgré tous les efforts déployés et alors que plusieurs centaines d'anticorps monoclonaux anti-Candida ont été obtenus, cet objectif paraît de plus en plus irréalisable, tout au moins en ce qui concerne la spécificité relative aux polysaccharides pariétaux. Aucune des spécificités annoncées lors des descriptions préliminaires d'anticorps monoclonaux $[9,10]$ n'a été confirmée par l'analyse d'un nombre important d'isolats sauvages. Cette constatation remet en question les bases de la spécificité antigénique des levures, établies à l'aide d'anticorps polyclonaux. Il faut cependant noter que les deux types de réactifs sont très différents, IgG de lapin dans le cas de polyclonaux et IgM murines dans celui des monoclonaux.

\section{La biologie cellulaire, les processus de glycosylation et de sécrétion}

Les anticorps monoclonaux sont de remarquables outils de recherche en biologie cellulaire, car ils permettent de suivre conjointement, en immunochimie, la biosynthèse des molécules portant l'épitope et, par immunodétection ultrastructurale, les compartiments cellulaires impliqués. L'anticorps monoclonal $5 \mathrm{~B} 2$, produit à la suite d'une infection expérimentale contre la phase pathogène de $C$. albicans, réagit avec la copule polysaccharidique de mannoprotéines excrétées dans un milieu de culture synthétique [11]. Ces mannoprotéines se présentent sous forme de traînées après électrophorèse en gel de polyacrylamide (figure $2 B$ ). Ce comportement particulier correspond à des copules polysaccharidiques de masses moléculaires variables associées à une même protéine. Lors de cultures en présence de tunicamycine, inhibiteur de la $\mathrm{N}$-glycosylation (figure 3-1), seules 


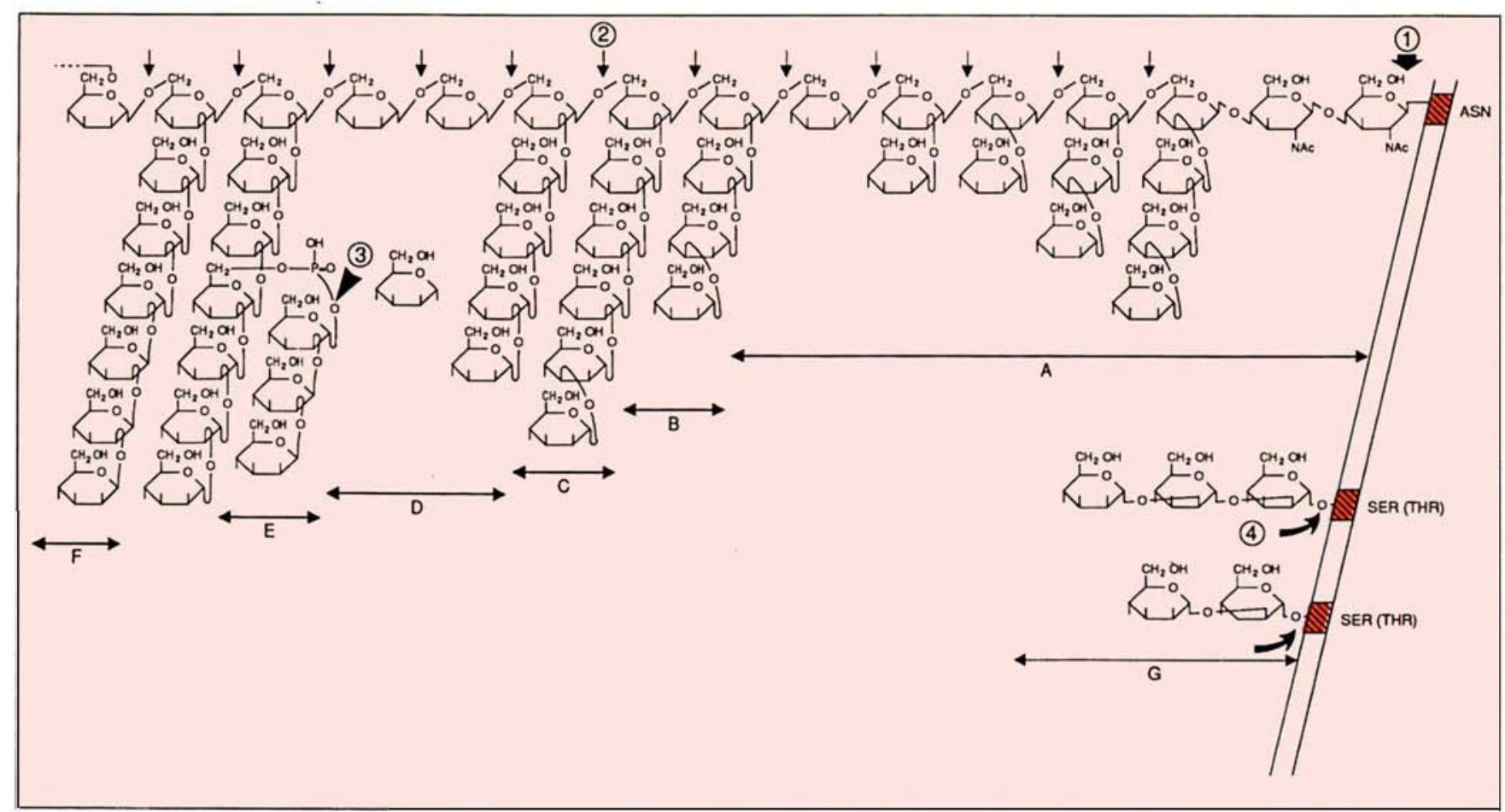

Figure 3. Schéma synthétique de l'organisation des phosphopeptidomannanes de la paroi de C. albicans. Deux types de liaisons relient les composés hydrocarbonés à la chaîne protéique. Les liaisons O-glycosidiques concernent de courtes chaînes oligosaccharidiques non ramifiées. Les liaisons $N$-glycosidiques concernent des édifices moléculaires plus complexes. Leur biosynthèse est inhibée par la tunicamycine (1). La chaîne interne (A) est une structure hautement conservée sur le plan phylogénétique, elle est en partie commune à tous les stades précoces de biosynthèse de glycoprotéines chez les cellules eucaryotes. Elle est ensuite différemment modifiée selon les espèces ou les tissus. Ces altérations intervenant dans l'appareil de Golgi ou ses équivalents fonctionnels concernent une dégradation plus ou moins complète et l'adjonction de divers résidus glucidiques. Chez les Candida en revanche, seuls des résidus mannose seront greffés sur cette structure de base relativement préservée. Le schéma classique fait référence à une chaîne linéaire formée de mannoses liés en $\alpha$ [1-6], sur laquelle sont greffées des chaînes latérales dont la taille varie de 1 à 7 mannoses reliés par des liaisons $\alpha$ [1-2] et $\alpha$ [1-3]. Le composé majoritaire chez C. albicans est un mannotétraose (B), un mannohexaose a été proposé comme étant le déterminant antigénique spécifique du sérotype $A$ de cette même espèce (C). Plus récemment, des ramifications des chaînes latérales ont été décrites (D), des liaisons $\beta$ [1-2], mises en évidence sur des oligomannosides liés par des ponts phosphodiester (E), enfin, l'existence de ces mêmes liaisons a été démontrée au sein de chaînes latérales non ramifiées (F). Les méthodes de coupure des phosphopeptidomannanes susceptibles d'aboutir à l'identification, au moins partielle, des épitopes oligosaccharidiques sont (1) la bêta-élimination (4), méthode d'hydrolyse ménagée en milieu alcalin affectant les liaisons O-glycosidiques; (2) I'hydrolyse acide ménagée (3) libérant les oligosaccharides liés par des ponts phosphodiesters ; (3) I'acétolyse ménagée (2) coupant préférentiellement les liaisons $\alpha$ [1-6].

les protéines dépourvues de copules polysaccharidiques portant les épitopes sont retrouvées dans le milieu (figure 2C). Le couplage direct de l'anticorps monoclonal à l'or colloïdal fournit une sonde très spécifique pour les réactions d'immunodétection ultrastructurale. Il est ainsi possible de localiser l'épitope dans des saccules et vésicules cytoplasmiques, à des stades vraisemblablement précoces de la biosynthèse des glycoprotéines [12], puis de suivre le processus de leur sécrétion au travers de la paroi de la levure. Les images obte$\mathrm{m} / \mathrm{s} n^{\circ} 6$ vol. 6 , juin 90 nues démontrent que cette sécrétion s'opère par des voies de cheminement préférentielles (figure 1B). Cette observation modifie les concepts classiques de l'organisation de la paroi fongique et pose la question fondamentale du ciblage transpariétal des glycoprotéines et de l'origine de l'énergie nécessaire à leur transport.

\section{Les épitopes oligosaccharidiques}

L'analyse rationnelle des informations relatives aux glycoprotéines identifiées par les anticorps monoclonaux implique l'identification des épitopes reconnus. De nombreuses difficultés sont encore rencontrées dans l'association des chémotypes oligosaccharidiques avec les sérotypes. Sur le plan chimique, il est montré que la structure des mannanes de $C$. albicans, longtemps considérée comme analogue à celle des mannanes de $S$. cerevisiae, est en fait beaucoup plus complexe. Des liaisons $\beta$ [1-2] ont été identifiées d'abord sur les oligosaccharides liés par des liaisons phosphodiesters [13] puis sur les chaînes 


\section{RÉFÉRENCES}

14. Shibata N, Fukasawa S, Kobayashi $H$, et al. Structural analysis of phospho-Dmannan-protein complexes isolated from ycast and mold form cells of Candida albicans NIH A-207 serotype A strain. Carbohydr Res 1989 ; 187 : 239-53.

15. Kogan G, Pavliak V, Masler L. Structural studies of mannans from the cell walls of the pathogenic yeasts Candida albicans serotypes A and B and Candida parapsilosis. Carbohydr Res 1988 ; 172 : 243-53.

16. Brawner D, Cutler J. Variability in expression of cell surface antigens of Candida albicans during morphogenesis. Infect Immun $1986 ; 51: 337-43$.

17. Lajean-Chaffin W, Skudlarck J, Morrow J. Variable expression of a surface determinant during proliferation of Candida albicans. Infect Immun 1988 ; 56 : 302-9.

18. Fruit J, Cailliez JC, Odds FC, Poulain D. Expression of an epitope by surface glycoproteins of Candida albicans. Variability among species, strains and ycast cells of the genus Candida. J Med Vet Mycol 1989 ; sous presse.

19. Brawner D, Cutler J. Oral Candida albicans isolates from non-hospitalized norma carriers, immunocompetent hospitalized patients, and immunocompromised patients with or without acquired immunodeficiency syndrome. J Clin Microbiol 1989; 27 : 1335-41.

20. Stlusky B, Buffo J, Soll D. Highfrequency switching of colony morphology in Candida albicans. Science $1985 ; 230$ : 666-9.

21. Strockbine NA, Largen MT, Zweibel SM, Buckley $\mathrm{H}$. Identification and molecular weight characterization of antigens from Candida albicans that are recognized by human sera. Infect Immun 1984; 43 715-21.

22. Strockbine NA, Largen MT, Buckley $\mathrm{H}$. Production and characterization of three monoclonal antibodies to Candida albicans proteins. Infect Immun 1984; 43 : 1012-8.

- Co-électrosynérèse : électrosynétèse sur acétale de cel. lulose impliquant des dépôts ponctuels, côte à côte, d'immunoglobulines, confrontés à un dépôt unique, linéaire, d'antigène. La coalescence des systèmes précipitants traduit l'identité des antigènes reconnus. latérales portées par la chaîne linéaire $\alpha[1-6,14]$. De même, l'existence de ramifications des mêmes chaînes latérales a été démontrée [15]. Toutes ces données sont schématisées sur la figure 3 où nous avons représenté les déterminants antigéniques oligosaccharidiques potentiellement présents sur une molécule de phosphopeptidomannane ainsi que les principes de leur séparation pour une analyse immunochimique.

Sur le plan immunochimique, la difficulté majeure réside dans la détermination de la spécificité des IgM monoclonales vis-à-vis d'oligosaccharides isolés. Les techniques de Farr d'inhibition en milieu liquide sont incompatibles avec le caractère multivalent de ces immunoglobulines et les techniques de chromatographie d'affinité mal adaptées. Les efforts se poursuivent pour trouver un support permettant une immunodétection sur produits séparés par chromatographie, notamment en réalisant des néoglycolipides. Il est d'autre part probable que l'affinité de l'anticorps risque de se manifester pour des structures voisines de celle de l'épitope ou encore que la conformation de celui-ci puisse être modifiée lors de sa séparation de son environnement naturel. La résolution de ces difficultés techniques, la compréhension des mécanismes de reconnaissance sont indispensables pour exploiter au mieux les nombreuses perspectives ouvertes par l'étude des anticorps monoclonaux dirigés contre des épitopes oligosaccharidiques. Ce problème déborde largement le cadre de la biologie des levures; il est probable que les levures en tant que source d'innombrables déterminants antigéniques naturels, faciles à produire et faciles à purifier, pourront aider à sa résolution.

\section{La variabilité antigénique}

Si les études sur la spécificité antigénique des levures ont été particulièrement décevantes, les anticorps monoclonaux se sont révélés en revanche de remarquables outils pour l'étude de la variabilité antigénique. Dès les premiers travaux, l'expression apparemment erratique des antigènes polysaccharidiques superficiels a été montrée comme étant une caractéris- tique essentielle [16]. Cette variabilité, qui se manifeste entre les souches d'une même espèce ainsi qu'entre les cellules d'une même souche au même stade morphologique, peut être le plus aisément objectivée en microscopie de fluorescence ou au cytofluorographe [17] et peut se manifester sous forme de secteurs au sein d'une colonie [18]. Il s'agit d'un caractère phénotypique, de tels secteurs pouvant apparaître au sein de colonies provenant de cultures monospores n'exprimant pas initialement l'antigène.

L'aptitude des anticorps monoclonaux à réagir de manière différente selon les souches d'une même espèce

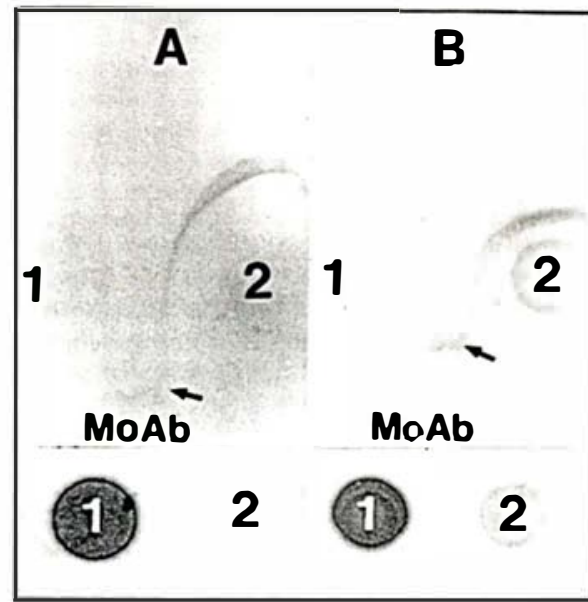

Figure 4. Évolution de l'antigénémie et de la réponse humorale correspondante chez deux patients $A$ et $B$ ayant présenté un épisode septicémique à C. albicans. Le premier sérum [1] prélevé au moment de l'épisode septicémique, présente en " immunodot " (bas de la figure) une réaction positive à l'égard d'un anticorps monoclonal anti-mannoprotéine de C. albicans, la présence de l'épitope n'est en revanche plus détectée dans le second sérum (2) prélevé alors que le patient traité est guéri. Les réactions de co-électrosynérèse * sur acétate de cellulose lhaut de la figure), réalisées afin de rechercher la présence $d^{\prime}$ anticorps anti-C. albicans dans les sérums 1 et 2, démontrent l'apparition de systèmes précipitants identifiant des molécules également reconnues par l'anticorps monoclonal (MoAb). Ces résultats signifient que les deux patients ont synthétisé des anticorps contre des molécules portant un épitope présent transitoirement dans leur sérum au cours de la phase septicémique. 
permet d'envisager leur utilisation en tant que marqueurs épidémiologiques. De fait, une étude vient de rapporter l'expression significativement plus faible d'un épitope identifié par un anticorps monoclonal chez les souches de sérotype $B$ déterminant des candidoses oro-pharyngées chez les sujets immunodéprimés [19]. Dans notre expérience, $10 \%$ des souches isolées en milieu hospitalier n'exprimaient pas l'épitope 5B2. Il est cependant remarquable qu'aucune des souches 5B2 négatives n'ait été isolée en position pathogène et que, à l'inverse, toutes les souches provenant de prélèvements pathologiques exprimaient l'épitope [18]. Il serait prématuré, à partir de ces deux exemples, de conclure à une relation de cause à effet, mais l'obtention d'un marqueur de comportement pathogène, même indirect, est d'un grand intérêt pour analyser le déterminisme de la pathogénicité. En ce sens, les monoclonaux sont des outils précieux. Encore faut-il comprendre le mécanisme moléculaire de la variabilité antigénique. Deux hypothèses sont actuellement envisageables concernant les épitopes oligosaccharidiques de surface. La première concerne la sécrétion discontinue des glycoprotéines porteuses, indépendante du cycle cellulaire. La seconde concerne un changement spontané du processus de glycosylation responsable de l'expression ou de la nonexpression de l'épitope sur une protéine dont la synthèse et la sécrétion sont continues. Ce processus pourrait résulter de l'expression de phénotypes alternatifs - ou " commutation " - [20] qui a déjà été proposée comme une base possible de l'adaptation parasitaire de C. albicans. Dans le cas présent, on comprend que l'expression spontanée d'épitopes différents au cours de la phase pathogène permettrait la sélection de levures pouvant proliférer sans être soumises à la pression immune de l'hôte.

\section{Le diagnostic biologique}

Deux types de méthodes sont susceptibles d'aider au diagnostic des candidoses profondes lorsque les méthodes mycologiques d'isolement et d'identification des levures sont en défaut. Il $s$ 'agit des méthodes de détection $\mathrm{m} / \mathrm{s} n^{\circ} 6$ vol. 6 , juin 90 d'anticorps et des méthodes de détection de substances fongiques circulantes. Les tests diagnostiques constituent, dans chacun de ces domaines, un compromis plus ou moins réussi entre facilité d'exécution, sensibilité et spécificité. Nous distinguerons les tests s'intéressant aux antigènes décrits classiquement comme protéiques et cytoplasmiques des tests relatifs aux glycoprotéines pariétales.

C'est à $\mathrm{H}$. Buckley et son équipe [21] que revient le mérite d'avoir initié les travaux sur les protéines cytoplasmiques (figure $2 A$ ) en postulant que la synthèse d'anticorps envers ces antigènes ne pouvait intervenir qu'après phagocytose, donc développement tissulaire des levures, alors que les anticorps anti-mannanes de la surface des levures présents dans le sérum de la majorité des individus sains pourraient ne résulter que du simple portage des levures. La poursuite d'une démarche rationnelle d'identification des antigènes protéiques cytoplasmiques à l'aide de sérums de patients [21], puis d'isolement et de caractérisation à l'aide d'anticorps monoclonaux [22], a conduit à décrire une famille d'antigènes cytoplasmiques de valeur diagnostique dont la masse moléculaire apparente se situe entre 45 et 52000 . Ultérieurement, de nombreuses équipes ont reconnu par la méthode du Western Blot, l'intérêt diagnostique de protéines cytoplasmiques de masse moléculaire voisine et donc très apparentées sinon identiques. Matthews et al. [23] ont récemment émis l'hypothèse que la présence d'anticorps dirigés contre une protéine de $47 \mathrm{kDa}$ serait non seulement très spécifique d'une candidose invasive mais traduirait la protection du patient. Il a pu être démontré, en outre, de manière non équivoque, par chromatographie d'affinité, que les antigènes $47 \mathrm{kDa}$ et $48 \mathrm{kDa}$ étaient présents dans les sérums de patients atteints de candidoses systémiques. Il est donc indéniable que ces antigènes présentent une grande valeur diagnostique; des tests commerciaux qui les concernent seront très prochainement disponibles. Les anticorps monoclonaux qui ont concouru à leur caractérisation $y$ seront impliqués - soit directement, soit en amont - lors des processus de purification. L'hypothèse d'une protection liée à la présence d'anticorps anti-47 kDa reste cependant à explorer avec son corollaire qui est l'utilité diagnostique de la détection d'anticorps liés à une protection.

La signification de la présence d'anticorps anti-mannanes est très controversée. Les techniques de routine du sérodiagnostic qui les détectent admettent un seuil significatif discriminant les sujets colonisés des sujets atteints de candidoses. Il est évident qu'un simple test permettra difficilement de différencier une réponse humorale résultant d'un accroissement de la colonisation au cours d'une hospitalisation prolongée d'un début d'invasion tissulaire, ou encore une fongémie d'une septicémie qui débute. Les anticorps monoclonaux ont cependant montré que "l'antigène mannane ", tel que le considèrent les biologistes médicaux, correspond en fait à une foule de déterminants antigéniques potentiels, exprimés différemment selon la biologie du Candida. Ces molécules, qui présentent la propriété de se fixer spécifiquement aux lymphocytes $\mathrm{T}$ [24], sont en outre des immunomodulateurs puissants [25]. Il est fort probable que, dans leurs interactions avec le système immunitaire humain, tous les épitopes synthétisés par la levure n'aient pas le même potentiel. La reconnaissance des bases moléculaires de ces interactions constitue pour nous un des défis les plus importants des recherches à conduire sur les Candida dans les prochaines années. Les monoclonaux spécifiques d'épitopes oligosaccharidiques y seront des outils indispensables. Une revue récente, dans ces mêmes colonnes [26], a déjà mentionné l'utilisation d'IgM monoclonales pour la détection d'antigènes circulants dans le sérum de patients, selon une méthode immunoenzymatique de type sandwich. Des " kits " commerciaux proposant une méthode d'agglutination de particules de latex sensibilisées également par des IgM monoclonales ont été récemment commercialisés [27]. Pour notre part, nous avons décrit une méthode d'" immunodot " sur nitrocellulose impliquant le même type de sonde couplée à l'or colloïdal (figure 4, p. 530), l'appréciation cinétique conjointe des profils d'antigénémie et de 


\section{RÉFÉRENCES}

23. Matthews $R$, Burnie J, Smith $D$, et al., Candida and AIDS : evidence for protective antibody. Lancet 1988 ; 8605 : 263-6.

24. Durandy A, Fisher A, Charron D, Griscelli C. Specific binding of antigens onto human $\mathrm{T}$ lymphocytes. $J$ Clin Invest $1986 ; 77: 1557-64$.

25. Domer J, Garner R, Befidi-Mengue R. Mannan as an antigen in cell-mediated immunity (CMI) assays and as a modulator of mannan-specific CMI. Infect Immun $1989 ; 57: 693-700$.

26. de Repentigny L, Boushira M. Diagnostic rapide des mycoses opportunistes par la détection d'antigènes circulants. medecine/sciences $1987 ; 3$ : 100-5

27. Georges E, Poirot JL, Garrigues ML, et al. Septicémie à Candida (Torulopsis) glabrata : détection du mannane sérique par un test au latex. Bull Soc Fr Mycol Med 1990 ; sous presse.

28. Poulain D, Ayadi A, Fruit J. Détection d'un antigène témoin d'infection systémique de Candida à l'aide d'un anticorps monoclonal couplé à l'or colloïdal. Ann Biol Clin 1988 ; 45 : 565-73.

29. Manning-Zweering $\mathrm{M}$, Maloney $\mathrm{C}$, Mitchell T, Weston H. Immunoblot analyses of Candida albicans-associated antigens and antibodies in human sera. $J$ Clin Microbiol 1986 ; 23 : 46-52.

30. Tojo M, Shibata $\mathbf{N}$, Kobayashi $\mathbf{M}$, Mikami T, Suzuki M, Suzuki S. Preparation of monoclonal antibodies reactive with beta-1, 2 linked oligomannosyl residues in the phosphomannan-protein complex of Candida albicans NIH B-792 strain. Clin Chem $1988 ; 34: 539-43$.

31. Fortier B, Hopwood V, Poulain D Electric and chemical fusions for the production of monoclonal antibodies reacting with the in vivo growth phase of Candida albicans. J Med Microbiol 1988 ; 27 : 239-45.

32. Casanova M, Gil ML, Cardenoso L, Martinez JP, Sentandreu R. Identification of wall-specific antigens synthesized during germ tube formation by Candida albicans. Infect Immun $1989 ; 57: 262-71$.

33. Sundstrom PM, Tam RM, Nichols EJ, Kenny GE. Antigenic differences in the surface mannoproteins of Candida albicans as revealed by monoclonal antibodies. Infect Immun 1988 ; 56 : 601-6. la réponse humorale vis-à-vis de mannanes permet d'établir des corrélations biocliniques relatives à l'évolution de l'infection ([28], figure 4). De nombreux obstacles restent encore à franchir, comme ceux relatifs à la réactivité croisée entre les glycoprotéines sériques et les anticorps antimannanes. La glycosylation des protéines est en effet un processus hautement conservé phylogénétiquement et les premières étapes de la biosynthèse des glycoprotéines humaines sont semblables à celles des levures (figure $3 A$ ). Les liaisons $\operatorname{Man} \alpha$ (1-2) et $\operatorname{Man} \alpha(1-3)$ sont très représentées au sein des glycoprotéines humaines. Nous pensons que ces structures constituent les supports les plus vraisemblables des réactions croisées entre glycoprotéines humaines et antisérums anti-Candida qui ont été révélées par Western Blot [29]. Notre propre expérience avec les anticorps monoclonaux anti-Candida a permis de démontrer un phénomène analogue. Il est probable que les interférences résultant de ces réactions croisées nuisent à la spécificité des tests de détection des mannanes circulants lorsque les sérums ne sont pas traités au préalable par la chaleur [26]. De façon à éviter l'incidence de ce phénomène, Tojo et al. [30] ont produit astucieusement, à des fins de détection de mannanes de Candida circulants, un anticorps monoclonal anti-Man $\beta$ (1-2) (figure $3 E$ ), structure non présente dans les glycoprotéines humaines.

Notons enfin que nombre de travaux ont concerné la recherche d'antigènes exprimés spécifiquement lors de la phase mycélienne, prédominante lors de l'invasion tissulaire alors que, la phase saprophytique prolifere très généralement sous forme levure. Les tests de détection d'anticorps vis-à-vis d'antigènes mycéliens ont été décrits à de nombreuses reprises comme plus spécifiques que ceux s'adressant aux antigènes de levures. De fait, nous avons pu obtenir un anticorps monoclonal ne réagissant in vitro qu'avec la surface des tubes germinatifs phase mycélienne primitive de $C$. albicans -, alors que in vivo, toutes les levures envahissant les tissus expriment massivement l'antigène correspondant, ce qui - a priori démontre la validité de cette démar- che [31]. D'autres anticorps monoclonaux, apparemment spécifiques de tubes germinatifs ont été décrits [32] et les antigènes mannoprotéiques reconnus, partiellement caractérisés [33]. Ces sondes sont potentiellement intéressantes, car elles s'adressent à des épitopes exprimés parallèlement à un phénomène morphogénétique, lui-même lié à la pathogénicité. Cependant, les travaux qui les concernent sont pour l'instant trop préliminaires sur le plan de la biologie cellulaire et de la physiopathologie pour pouvoir conclure.

L'ensemble des perspectives ouvertes dans le domaine du diagnostic sérologique est prometteur. Cependant notre expérience diagnostique nous a montré qu'en raison de la nature opportuniste de l'agent infectieux, il est illusoire de penser qu'un simple test permettra de porter le diagnostic et de prévoir l'évolution de l'infection. Quelles que soient les performances des tests à venir, l'application cinétique de méthodes de détection d'anticorps et/ou d'antigènes, susceptibles de fournir respectivement une évaluation indirecte et directe d'une prolifération fongique tissulaire, restera indispensable.

\section{Le diagnostic paraclinique}

Les anticorps monoclonaux radiomarqués sont utilisés depuis quelques années pour la localisation de tumeurs humaines en imagerie médicale. Ces méthodes présentent des indications évidentes pour la localisation des foyers profonds candidosiques, cardiaques, rénaux, vésiculaires..., dont nous avons vu que le diagnostic était extrêmement difficile et qui, souvent, sont malheureusement des découvertes nécropsiques ou des surprises opératoires. Les études que nous avons conduites chez le cobaye avec un anticorps monoclonal anti-C. albicans marqué à l'iode 131 démontrent qu'il est possible de relier le niveau de parasitisme d'un organe - apprécié par rétroculture* - et la fixation de radioactivité appréciée par

\footnotetext{
* Mise en culture d'organes broyés permettant de déterminer le nombre d'unités formant colonie par gramme d'organe.
} 

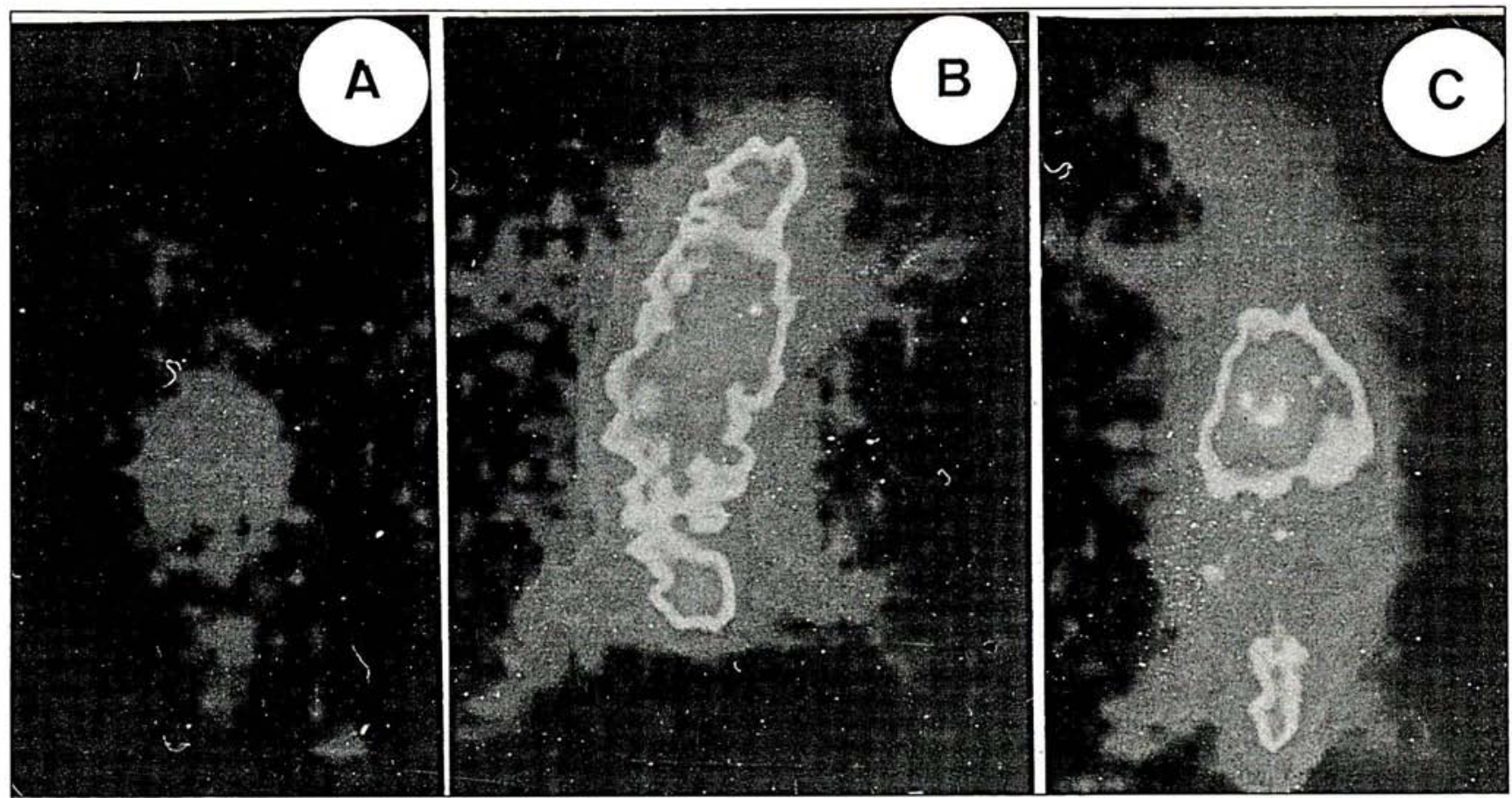

Figure 5. Détection gammagraphique de foyers prolifératifs candidosiques in vivo. Images obtenues $24 \mathrm{~h}$ après l'injection d'un anticorps monoclonal antimannoprotéines de C. albicans marqué à l'iode 131. A, animal témoin non infecté. $B$ et $C$, animaux infectés par injection intraveineuse de levures. L'animal $A$ fait l'objet d'un marquage très faible, les rétrocultures effectuées après autopsie ont montré que l'animal $B$, dont la majorité des viscères sont marqués, a fait l'objet d'une infection disséminée affectant le massif facial, le thorax et l'abdomen. L'animal C a développé une infection plus localisée avec, en particulier, un hyperparasitisme vésiculaire parfaitement objectivable sur la gammagraphie.

scintigraphie (figure 5). Ces résultats encourageants permettent d'entrevoir une application clinique qui sera peutêtre d'interprétation moins délicate que pour les tumeurs, grâce à une discrimination immunologique plus aisée des foyers prolifératifs fongiques par rapport aux tissus environnants.

\section{Conclusion, perspectives}

Les anticorps monoclonaux antiCandida ont donc fourni des sondes diagnostiques qui seront d'un usage courant. Sur le plan fondamental, ils ont permis de préciser les connaissances sur les glycoprotéines de surface et de démontrer de manière non équivoque leur variabilité antigénique, longtemps suspectée comme un moyen d'adaptation constante des levures à la pression immunitaire du milieu. Les bases moléculaires de cette variabilité concernent très vraisemblablement l'expression d'épitopes oligosaccharidiques dont les interactions avec le système immunitaire de l'hôte sont désormais essentielles à définir

$m / s n^{\circ} 6$ vol. 6 , juin 90

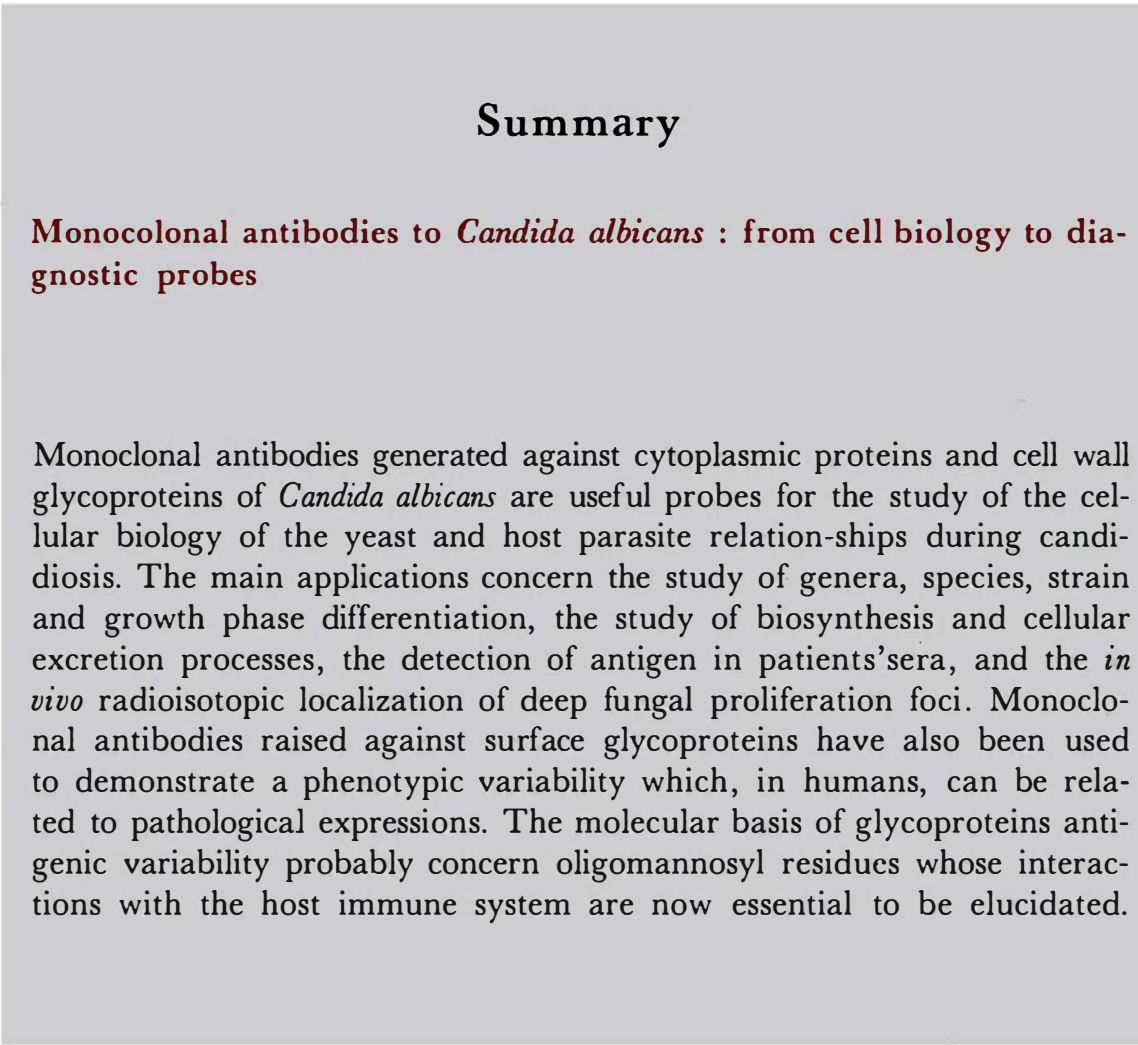

Monoclonal antibodies generated against cytoplasmic proteins and cell wall glycoproteins of Candida albicans are useful probes for the study of the cellular biology of the yeast and host parasite relation-ships during candidiosis. The main applications concern the study of genera, species, strain and growth phase differentiation, the study of biosynthesis and cellular excretion processes, the detection of antigen in patients'sera, and the in vivo radioisotopic localization of deep fungal proliferation foci. Monoclonal antibodies raised against surface glycoproteins have also been used to demonstrate a phenotypic variability which, in humans, can be related to pathological expressions. The molecular basis of glycoproteins antigenic variability probably concern oligomannosyl residues whose interactions with the host immune system are now essential to be elucidated. 